\title{
Neuropilin-1 is associated with clinicopathology of gastric cancer and contributes to cell proliferation and migration as multifunctional co-receptors
}

Linhao Li ${ }^{1}$, Xian Jiang ${ }^{2}$, Qian Zhang ${ }^{3}$, Xuesong Dong ${ }^{2}$, Yuqiang Gao ${ }^{4}$, Yuanlong He ${ }^{4}$, Haiquan Qiao ${ }^{2}$, Fangyu Xie ${ }^{4}$, Xiangjun $\mathrm{Xie}^{4^{*}}$ and Xueying Sun ${ }^{2^{*}}$

\begin{abstract}
Background: Neuropilin-1 (NRP-1) is a transmembrane glycoprotein participating in the growth and metastasis of cancer cells as multifunctional co-receptors by interacting with the signaling pathways. However, its role in gastric cancer has not yet been clarified. This study aims to investigate whether NRP-1 expression is associated with the clinicopathology of gastric cancer, and involved in the growth and metastasis of gastric cancer cells.

Methods: NRP-1 expression in clinical gastric cancer specimens was examined by immunohistochemistry and its association with clinicopathology analyzed. The expression of NRP-1 in a panel of human gastric cancer cells was examined by real-time RT-PCR and immunoblotting. Stable transfectants depleted of NRP-1, termed MGC-803-NRP ${ }^{\text {low }}$, were generated from MGC-803 cells. Cell proliferation was analyzed by the Cell Counting Kit-8 and Bromodeoxyuridine incorporation assays, and migrating ability analyzed by migration assays. The xenograft model was used to assess the effects of NRP-1 depletion on tumorigenesis, growth, metastasis and therapeutic potentials. The role of NRP-1 as co-receptors in the signaling pathways stimulated by ligands was examined. The key molecules involved in cell proliferation, migration and related signaling pathways were detected by immunoblotting.
\end{abstract}

Results: Gastric cancer tissues expressed higher levels of NRP-1 compared to normal gastric mucosa. Its expression correlated with clinical staging, tumor differentiation and pathological types. NRP-1 depletion inhibited cell proliferation by inducing cell cycle arrest in the G1/S phase by upregulating p27, and downregulating cyclin E and cyclin-dependent kinase 2. NRP-1 depletion reduced the ability of cells to migrate by inhibiting the phosphorylation of focal adhesion kinase. NRP-1 depletion suppressed tumorigenesis, tumor growth and lung metastasis by inhibiting cell proliferation and tumor angiogenesis in situ. Therapeutic NRP-1 shRNA inhibited the growth of established BGC823 tumors. Depletion of NRP-1 inhibited the activation of VEGFNEGFR2, EGF/EGFR and HGF/C-Met pathways stimulated by respective recombinant human VEGF-165, EGF and HGF proteins.

Conclusions: The present results indicate that NRP-1 may be a potentially valuable biomarker and therapeutic target for gastric cancer.

Keywords: Neuropilin-1, Gastric cancer, Clinicopathology, Proliferation, Metastasis, Cellular signaling

\footnotetext{
*Correspondence: xiangjunxie2005@126.com; kevsun88@hotmail.com

${ }^{4}$ Department of Gastroenterology, Qingdao Municipal Hospital, No. 1

Jiaozhou Road, Qingdao 266011, China

${ }^{2}$ Department of General Surgery, the First Affiliated Hospital of Harbin

Medical University, No. 23 Youzheng Street, Harbin 150001, China

Full list of author information is available at the end of the article
} 


\section{Background}

Neuropilin-1 (NRP-1), a transmembrane glycoprotein, was initially identified as a neuronal receptor for the class 3 semaphorins [1]. It is now known that NRP-1 also acts as multifunctional co-receptors participating in the initiation, growth and metastasis of cancer cells [1]. NRP-1 is overexpressed in several common types of cancer. More importantly, NRP-1 expression associates with the advanced stage of disease, and negatively correlates with the prognosis of hepatocellular carcinoma [2], colorectal cancer [3], and esophagus squamous cell carcinoma [4], indicating that NRP-1 is a potential molecular target for cancer therapy. Specific inhibition of NRP-1 has been shown to suppress the growth and metastasis of several types of cancer cells [5-9].

The mechanisms for the role of NRP-1 in cancer progression rely on its interactions with several key signaling pathways in cancer cells. NRP-1 interacts with vascular endothelial growth factor (VEGF) and its receptor VEGFR2, leading to the activation of this pathway and promoting tumor angiogenesis and growth [10-12]. NRP-1 also co-interacts with other important heparinbinding cytokines, such as epithelial growth factor (EGF) and its receptor (EGFR) [13], and hepatocyte growth factor (HGF) and its receptor c-Met [14], and activates these two pathways. The above three cellular signaling pathways are all shown to be involved in the progression of gastric cancer [15-18].

It has been reported that NRP-1 is expressed in human gastric cancer cells and in limited number of human gastric cancer tissues [15]. However, the role of NRP-1 in the progression of gastric cancer and the related mechanisms has not been fully elucidated. Gastric cancer is the third most common cause of cancer-related death in men worldwide [19]. Conventional adjuvant treatments have shown only modest effects on the survival of patients with advanced gastric cancer, and the development of molecular targeting drugs for gastric cancer is lagging behind some other cancers [20]. Therefore, it is essential for seeking novel molecular targets for combating gastric cancer. Here, we designed the present study aiming to investigate whether NRP-1 expression correlates with the clinicopathologic features of gastric cancer, and whether NRP-1 depletion could inhibit the growth and metastasis of gastric cancer cells, and whether NRP-1 displays its role as co-receptors by interacting the VEGF, EGF and HGFmediated pathways in gastric cancer cells.

\section{Methods}

\section{Patients}

A total of 141 consecutive patients with pathologically proved gastric cancer received curative resection at the Department of General Surgery, Qingdao Municipal Hospital in China, between January, 2011 and December,
2013. None of the patients received any preoperative anticancer treatments. Gastric cancer was staged according to the staging system by the American Joint Committee on Cancer [21]. The histologic classification of gastric carcinoma was performed according to the 2010 WHO histologic classification system and cell differentiation [21]. This study was approved by the Ethics Committee of Qingdao Municipal Hospital (Qingdao, China), and all patients gave their informed consent prior to the inclusion in the study.

\section{Cell culture, antibodies and reagents}

Human gastric cancer cells MGC-803, SGC7901, BGC823, AGS, NCI-N87 and HGC-27 were obtained from the Type Culture Collection Cell Bank (Chinese Academy of Sciences Committee, Shanghai, China). Cells were cultured in RPMI-1640 medium supplemented with $10 \%$ FBS (fetal bovine serum) at $37{ }^{\circ} \mathrm{C}$ in a humidified atmosphere of $5 \%$ $\mathrm{CO}_{2}$. For antibodies (Abs) and reagents, please refer to Additional file 1.

\section{Immunohistochemistry of clinical specimens}

Formalin-fixed specimens were transferred to $70 \%$ ethanol, and subsequently paraffin-embedded, sectioned and mounted on 3-aminopropyltriethoxysilane-coated slides (Sigma, Shanghai, China). Antigen retrieval was performed by heating sections in a microwave in $0.01 \mathrm{M}$ citrate buffer. Sections were blocked for $2 \mathrm{~h}$, and incubated with a rabbit anti-human NRP-1 Ab (diluted 1:250) at $4{ }^{\circ} \mathrm{C}$ overnight. A standard horseradish peroxidase staining procedure was followed using a biotinylated secondary Ab (diluted 1:250, and immunoreactivity developed with Sigma FAST DAB (3,3'-diaminobenzidine tetrahydrochloride) and $\mathrm{CoCl}_{2}$ enhancer tablets. Sections were counterstained with hematoxylin. Normal rabbit sera were diluted 1:10 in PBS, and used for blocking and dilution of Abs. Negative controls were achieved by using irrelevant goat IgG at a dilution of 1:50. NRP-1 staining was assessed in 20 randomly selected fields per specimen using a semi-quantitative grading system, which reflected the proportion and intensity of staining present within the specimen. The staining intensity (Value A) was graded in a four-tier grading system: no staining (0), faint yellow (1), yellow (2) and brown (3). The extent of positive staining (Value B) was determined using a four-tier grading system based on the percentage of positive cells: $\leq 10 \%(1), 11-40 \%(2), 41-70 \%$ (3), and $\geq 70 \%$ (5). The immunohistological score for each specimen was calculated by $\mathrm{A} \times \mathrm{B}$, and then each specimen was graded lower level $(\leq 5)$ and higher level $(>5)$.

\section{ShRNA expression vectors}

The sequence targeting NRP-1 (GAGAGGUCCUGAAUG UUCC), corresponding to nucleotides 949-967 of human NRP-1 (GenBank NM_003873.5), was previously reported 
[13]. The chemically synthesized oligonucleotides were introduced into the pSuppressorNeo vector to generate NRP-1 shRNA as described previously [22, 23]. A scrambled shRNA vector (Sc-shRNA) targeting nonspecific sequence (UUCUCCGAACGUGUCACGU) served as controls.

\section{Establishment of stable transfectants, Cell viability} analysis, Bromodeoxyuridine (BrdU) incorporation assay, Assessment of cell cycle, Transfection of siRNA, Quantitative reverse-transcription polymerase chain reaction (qRT-PCR), Migration assay, Immunoblotting, In situ Ki-67 proliferation index and Assessment of tumor vascularity

Detailed methods have been described previously [22, 23] and can be found in Additional file 1.

\section{Animal experiments}

Six to 8-week-old male nude BALB/c mice ( $\mathrm{H}-2 \mathrm{~b})$ were obtained from the Animal Research Center, The First
Affiliated Hospital of Harbin Medical University, China. The experimental protocol was described previously [22, 23]. This study was approved (permit SYXK20020009) by the Animal Ethics Committee of Harbin Medical University (Harbin, China).

\section{Tumorigenicity study}

Cells $\left(2 \times 10^{6}\right)$ were injected subcutaneously into groups of 10 mice. The animals were monitored every 3 days. Mice were sacrificed 24 days later, and tumors were harvested and weighed.

\section{In vivo metastasis study}

Cells $\left(1 \times 10^{6}\right)$ were injected into groups of 8 mice via the tail vein. The mice were sacrificed 7 weeks later. Lungs were harvested, weighed, then sectioned and stained by hematoxylin/eosin.

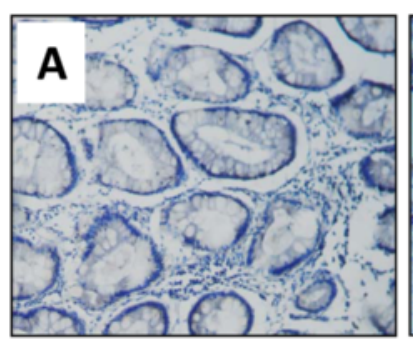

D

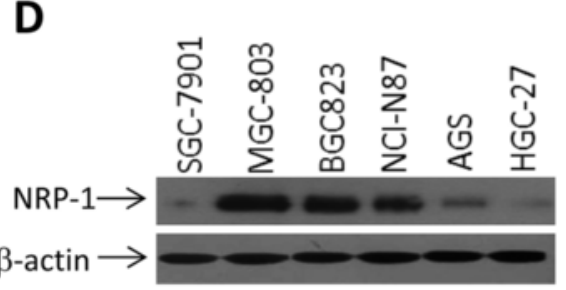

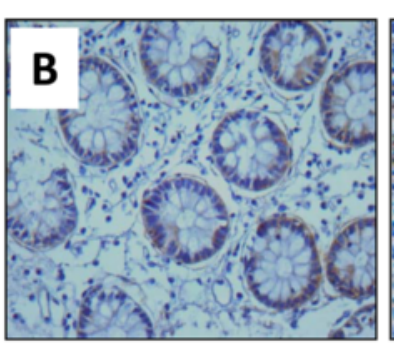

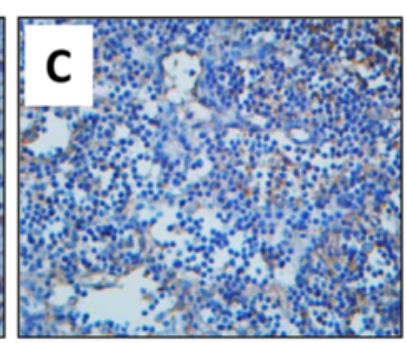

E

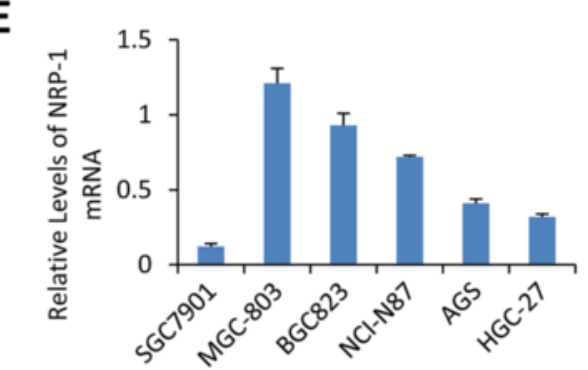

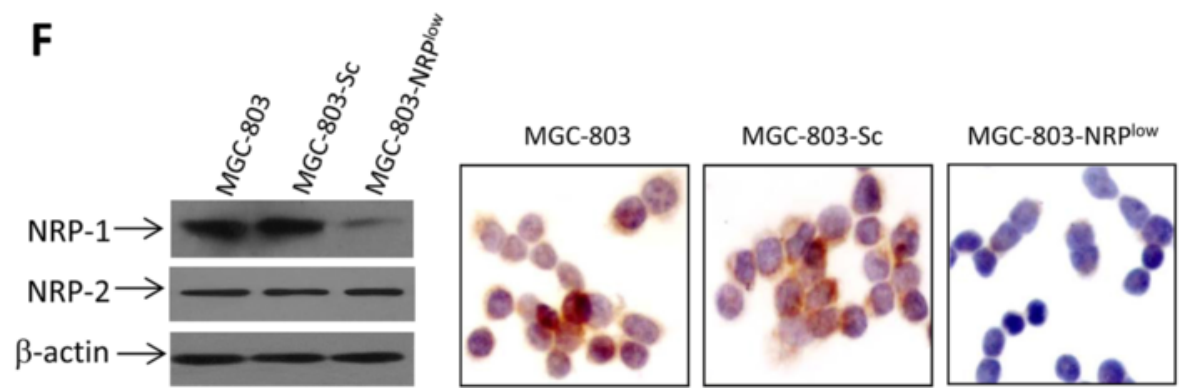

Fig. 1 Expression of NRP-1 in gastric cancer tissues and cells. a-c Representative images of section from gastric normal mucosa (a), adenocarcinoma (b) and undifferentiated cancer (c), immunostained with an anti-NRP-1 antibody. $\mathbf{d}$ Lysates of human gastric cancer cells were immunoblotted. e The expression of NRP-1 mRNA was analyzed by qRT-PCR, and normalized to $\beta$-actin. $\mathbf{f}$ The expression of NRP-1 and NRP-2 was examined in lysates of parental MGC-803, MGC-803-SC and MGC-803-SUR ${ }^{\text {low }}$ cells by immunoblotting. NRP-1 expression in the three cell lines was further detected by immunohistochemistry 


\section{Therapeutic effect study}

Cells $\left(5 \times 10^{6}\right)$ were injected subcutaneously into the flanks of mice. When tumors reached $\sim 100 \mathrm{~mm}^{3}$, the mice were assigned to 2 groups of 8 mice, which received an intratumoral injection of Sc-shRNA or NRP1 shRNA, respectively. The shRNA transfection solution was prepared by mixing shRNA vector, Lipofectamine2000 and serum-free medium. Each tumor received an injection of $50 \mu \mathrm{l}$ shRNA transfection solution containing $200 \mu \mathrm{g}$ shRNA. Two mice from each group were sacrificed 4 days after intratumoral injection for detecting the expression of NRP-1. Tumors were measured and mice killed 20 days later.

\section{Statistical analysis}

Association between NRP-1 expression and clinicopathologic parameters was analyzed by Mann Whitey test. Other data are expressed as mean values \pm standard deviation. Comparisons were made with a one-way analysis of variance (ANOVA) followed by a Dunnet's test. $P<0.05$ was considered statistically significant.

\section{Results}

\section{NRP-1 expression in gastric cancer tissues and its} correlation with clinicopathologic parameters

Normal gastric mucosa had weak NRP-1 expression (Fig. 1a), whereas gastric cancer tissues expressed different levels of NRP-1 protein (Table 1). Representatively, gastric adenocarcinoma (Fig. 1b) expressed relatively lower levels, while undifferentiated gastric cancer (Fig. 1c), relatively higher levels of NRP-1. The level of NRP-1 expression was significantly correlated with clinical staging, tumor differentiation and pathological types of gastric cancer, but not with patients' gender and age (Table 1).

\section{NRP-1 expression in gastric cancer cell lines}

Human gastric cancer cells expressed different levels of NRP-1 protein (Fig. 1d), and mRNA (Fig. 1e). MGC-803 cells expressing the highest level of NRP-1 were selected for generating stable transfectants. MGC-803 cells stably transfected with NRP-1 shRNA or Sc-shRNA were termed MGC-803-NRP ${ }^{\text {low }}$ and MGC-803-Sc, respectively (Fig. 1f). Compared with parental MGC-803 cells, MGC-803NRP ${ }^{\text {low }}$ cells expressed significantly lower, while MGC-803Sc cells expressed similar, levels of NRP-1. In addition, the three cell lines expressed similar levels of NRP-2 (Fig. 1f).

\section{NRP-1 depletion inhibits cell growth}

MGC-803-NRP ${ }^{\text {low }}$ cells had significantly lower viability, while MGC-803-Sc cells had the similar viability, compared with parental MGC-803 cells (Fig. 2a). We examined the expression of cyclin E, CDK2 and p27, which play key roles in cell proliferation and cycle progression [24]. The results showed that MGC-803-NRP ${ }^{\text {low }}$
Table 1 Relationship between NRP-1 protein expression and clinical pathological parameters

\begin{tabular}{|c|c|c|c|c|}
\hline & Number & $\begin{array}{l}\text { Lower level } \\
\text { of NRP-1 }\end{array}$ & $\begin{array}{l}\text { Higher level } \\
\text { of NRP-1 }\end{array}$ & $P$ value \\
\hline Gender & & & & 0.738 \\
\hline Male & 87 & 47 & 40 & \\
\hline Female & 54 & 25 & 29 & \\
\hline $\begin{array}{l}\text { Age median (range): } \\
53.4 \text { (27-82) }\end{array}$ & & & & 0.345 \\
\hline$<60$ & 93 & 43 & 50 & \\
\hline$\geq 60$ & 48 & 29 & 19 & \\
\hline TNM stage & & & & 0.019 \\
\hline Stage 0 & 5 & 4 & 1 & \\
\hline Stage I & 26 & 20 & 6 & \\
\hline Stage ॥ & 38 & 23 & 15 & \\
\hline Stage III & 49 & 18 & 31 & \\
\hline Stage IV & 23 & 7 & 16 & \\
\hline $\begin{array}{l}\text { WHO histologic } \\
\text { classification }\end{array}$ & & & & 0.046 \\
\hline Tubular & 67 & 38 & 29 & \\
\hline Papillary & 42 & 22 & 20 & \\
\hline Mucinous & 13 & 4 & 9 & \\
\hline Poorly cohesive & 11 & 3 & 8 & \\
\hline $\begin{array}{l}\text { Uncommon histologic } \\
\text { variants }\end{array}$ & 8 & 5 & 3 & \\
\hline Differentiation & & & & 0.025 \\
\hline Well & 35 & 27 & 8 & \\
\hline Moderate & 74 & 36 & 38 & \\
\hline Poor & 32 & 9 & 23 & \\
\hline
\end{tabular}

Notes: NRP-1, Neuropilin-1. P value was estimated by Mann Whitney test

cells expressed significantly lower levels of cyclin $\mathrm{E}$ and CDK2, and a higher level of p27, than parental MGC-803 cells (Fig. 2b). However, MGC-803-Sc cells expressed similar levels of the above proteins compared with parental MGC-803 cells (Fig. 2b). We then examined cell proliferation by means of BrdU-DAPI staining (Fig. 2c, d). MGC-803-NRP ${ }^{\text {low }}$ cells had a significantly lower rate of proliferation than parental MGC-803 cells. Specifically, $58.5 \pm 7.3 \%$ of parental MGC-803 cells, while only $19.6 \pm 3.8 \%$ of MGC-803-NRP ${ }^{\text {low }}$, were BrdUpositive (Fig. 2c, d). We further detected cell cycle distribution by flow cytometry. The fraction of cells at the G1 phase was significantly higher in MGC-803-NRP ${ }^{\text {low }}$ cells compared with parental MGC-803 cells (Fig. 2e, f).

\section{NRP-1 depletion inhibits cell migration}

The number of migrated MGC-803-NRP ${ }^{\text {low }}$ cells was significantly lower than that of parental MGC-803 cells (Fig. 3a, b). It has been reported that NRP-1 interacts the VEGF/VEGFR2 pathway, resulting in phosphorylation of 


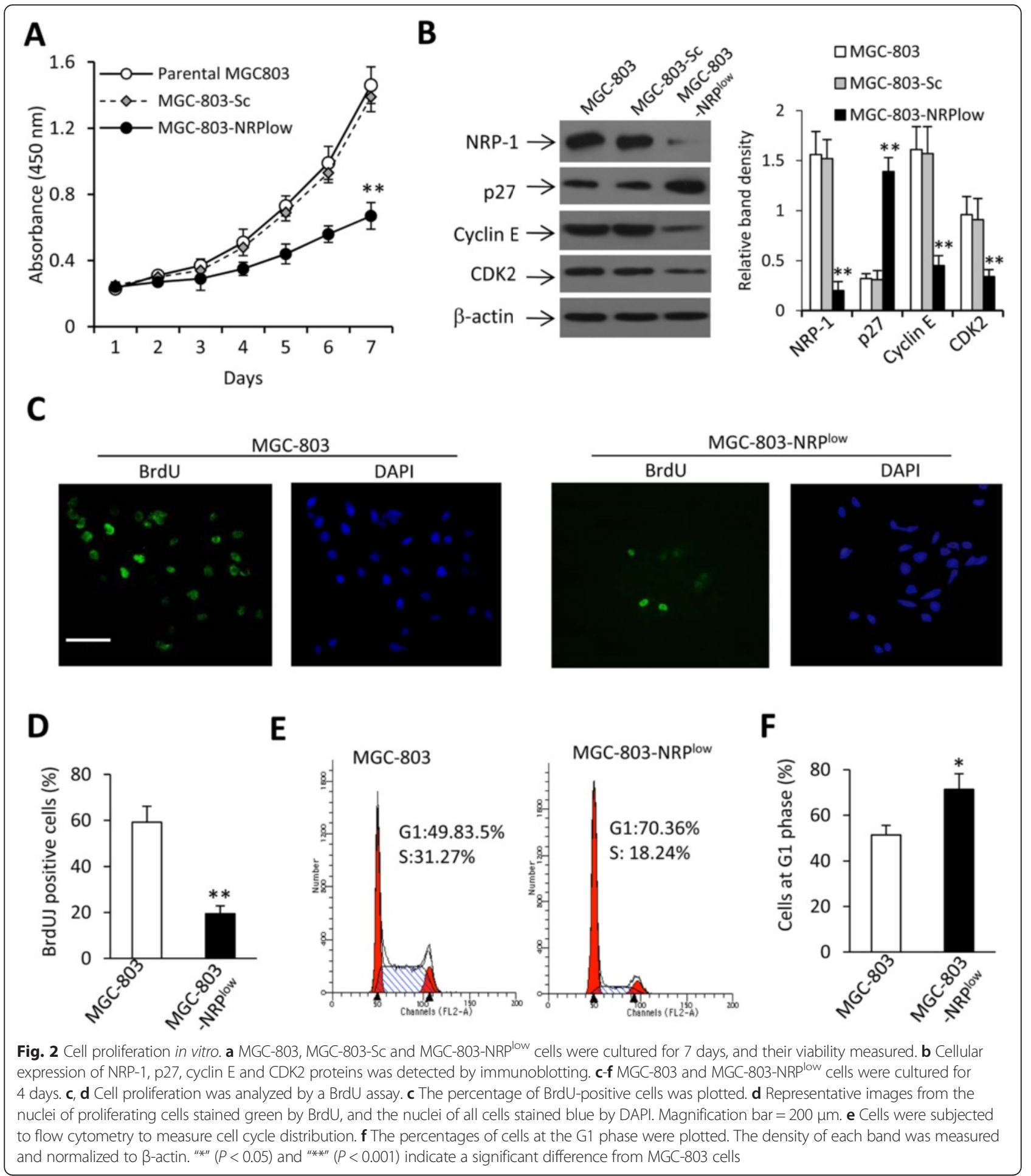

FAK, a key factor for cell migration [11]. Here we showed that MGC-803-NRP ${ }^{\text {low }}$ cells expressed lower, but MGC803-Sc cells expressed similar, levels of P-FAK, compared with parental MGC-803 cells (Fig. 3c). The three cell lines expressed similar levels of FAK (Fig. 3c).
NRP-1 depletion inhibits tumorigenesis, tumor growth and metastasis

Based on the in vitro results, we further investigated the effects of NRP-1 in animal models. Since MGC-803-Sc cells did not show significant different from parental 
A

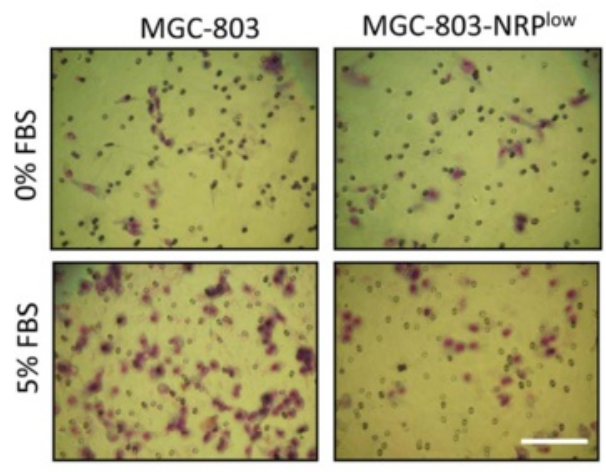

B
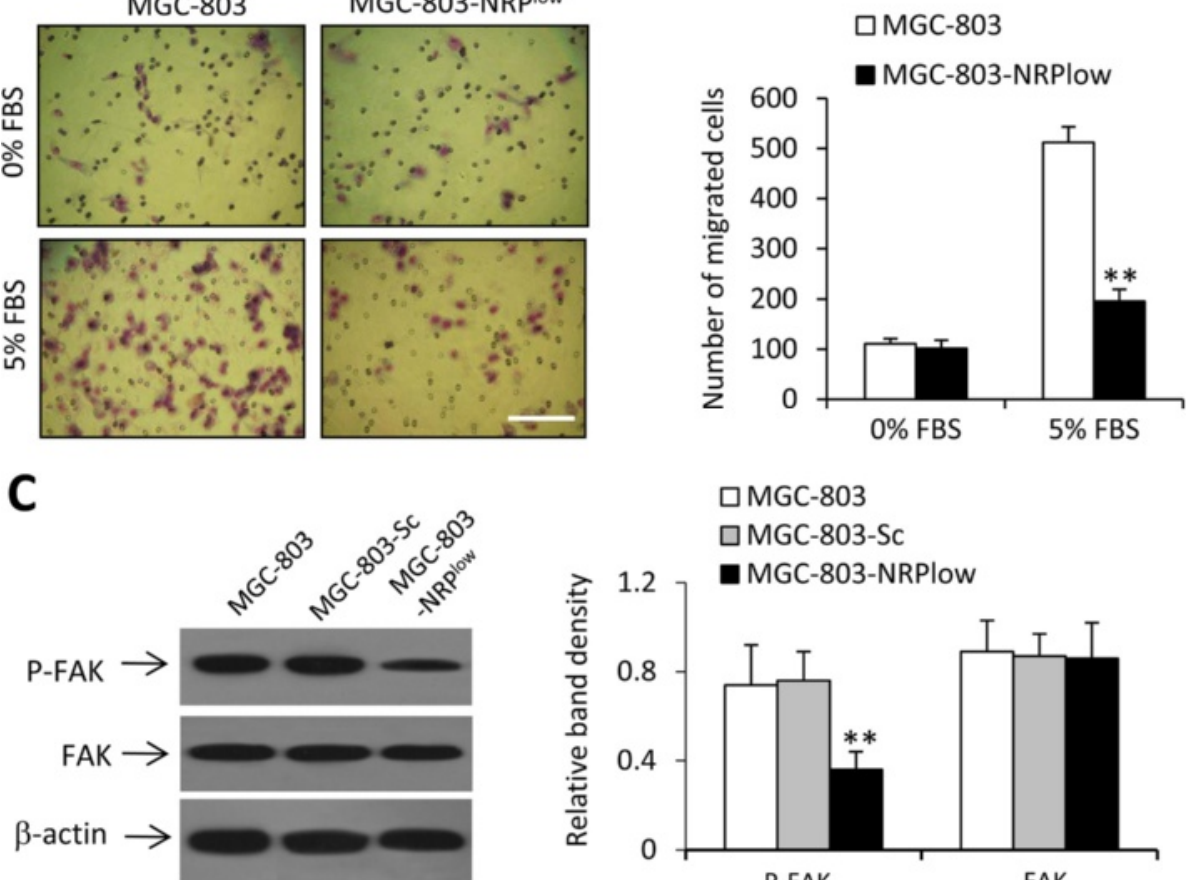

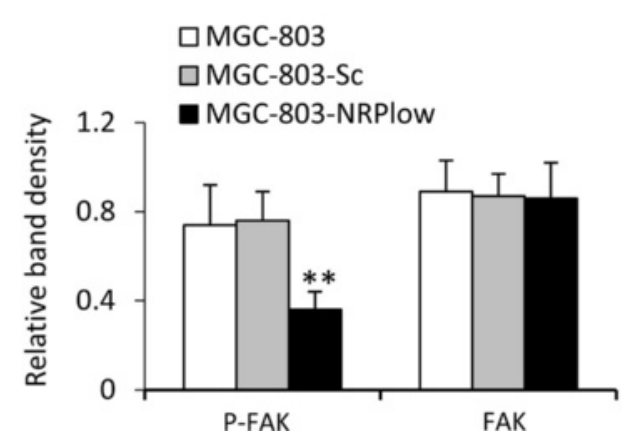

Fig. 3 Cell migration. a, b MGC-803 and MGC-803-NRPlow cells were subjected to migration assays, in the presence or absence of $5 \%$ FBS as chemoattractants. $\mathbf{a}$ Migrated cells were visualized using Giemsa staining. Magnification bar $=200 \mu \mathrm{m}$. b Numbers of migrating cells were counted. c MGC-803, MGC-803-SC and MGC-803-NRP ${ }^{\text {low }}$ cells were immunoblotted to determine the expression of FAK and P-FAK. The density of each band was measured and normalized to $\beta$-actin. " ${ }^{* * * \prime \prime}(P<0.001)$ indicates a significant difference from parental MGC-803 cells

cells in expression of the above proteins involved in cell proliferation and migration, we only used parental MGC-803 cells as controls in the following experiments. As shown in Fig. 4a, subcutaneous tumors were detected in 9 out of 10 mice injected with parental MGC-803 cells, but in only 5 out of 10 mice receiving MGC-803-NRP ${ }^{\text {low }}$ cells. In addition, MGC-803-NRP ${ }^{\text {low }}$ tumors grew to $761.8 \pm 104.3 \mathrm{~mm}^{3}$ (1432.1 $\pm 209.6 \mathrm{mg}$ in weight), whereas control MGC-803 tumors reached $1722.4 \pm 89.6 \mathrm{~mm}^{3}$ (633.1 $\pm 90.7 \mathrm{mg}$ in weight), 24 days following cell inoculation (Fig. 4a, b). The mice injected with MGC-803-NRP ${ }^{\text {low }}$ cells had fewer and smaller metastatic nodules in their lungs (with an average weight of $235.7 \pm 18.5 \mathrm{mg}$ ), compared with those injected with parental cells (with an average weight $378.1 \pm 40.3 \mathrm{mg}$ ) (Fig. 4c, d). In the study for testing the therapeutic effects, we established subcutaneous tumors by using another gastric cancer cell line, BGC823, which was also shown to express higher levels of NRP-1 (Fig. 1d, e). When tumors reached $100 \mathrm{~mm}^{3}$, they were injected with either Sc-shRNA or NRP-1 shRNA. As shown in Fig. 4e, intratumoral delivery of NRP-1 shRNA resulted in downregulation of NRP-1 in situ, compared with Sc-shRNA. BGC823 tumors treated with NRP-1 shRNA were significantly smaller $\left(769.1 \pm 82.4 \mathrm{~mm}^{3}\right)$ than those treated with Sc-
shRNA $\left(1521.7 \pm 118.5 \mathrm{~mm}^{3}\right), 20$ days after treatment started (Fig. 4f).

\section{NRP-1 depletion inhibits cell proliferation in situ and tumor angiogenesis}

The tumors harvested in Fig. 4b were immunostained for examining cell proliferation and tumor angiogenesis. In consistence with the in vitro results (Fig. 1f), MGC-803-NRP ${ }^{\text {low }}$ tumors expressed markedly lower levels of NRP-1 proteins than MGC-803 tumors (Fig. 5a). NRP-1 depletion significantly inhibited cell proliferation and tumoral angiogenesis (Fig. 5a-c). The expression of key molecules in vivo (Fig. 5d) was consistent with that obtained in vitro.

\section{NRP-1 depletion inhibits VEGF-, EGF- and HGF-activated pathways}

NRP-1 is shown to participate as co-receptors [10-14] in the activation of the VEGF/VEGFR2, EGF/EGFR and HGF/c-Met pathways, which play key roles in the proliferation and metastasis of gastric cancer cells $[17,18,25]$. Therefore, we investigated whether depletion of NRP-1 could suppress the activation of these pathways stimulated by respective ligands in gastric cancer cells. MGC803 and MGC-803-NRP ${ }^{\text {low }}$ cells were transfected with 
A

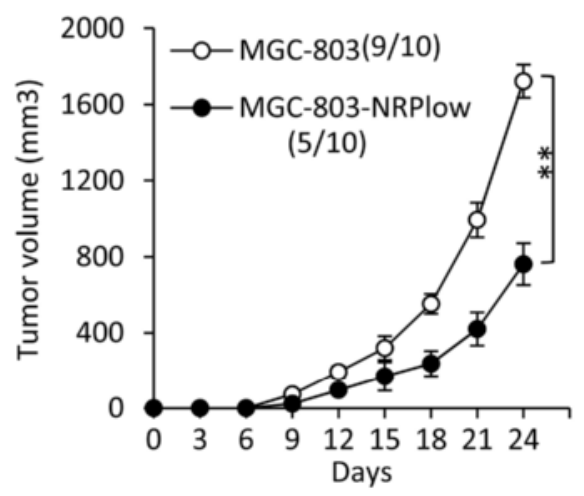

C

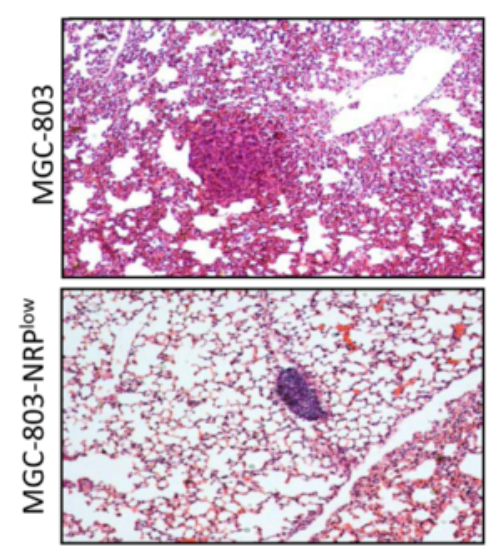

E

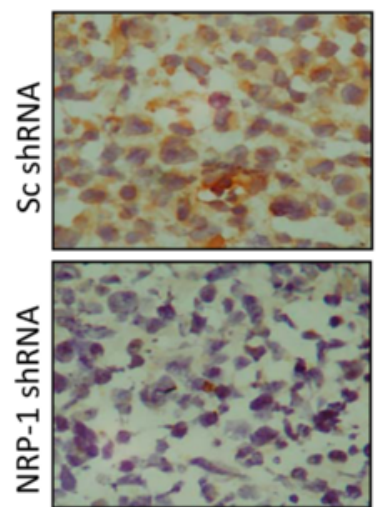

B

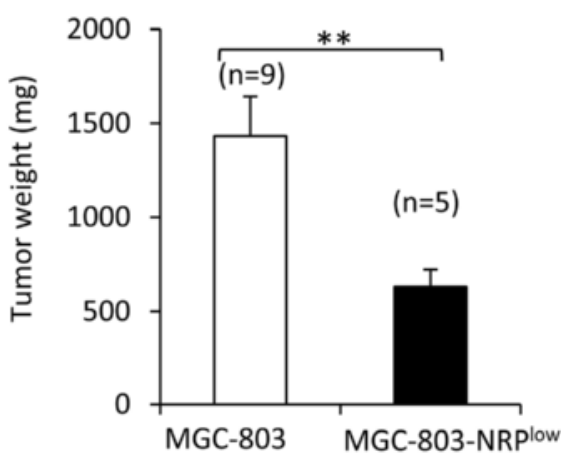

D

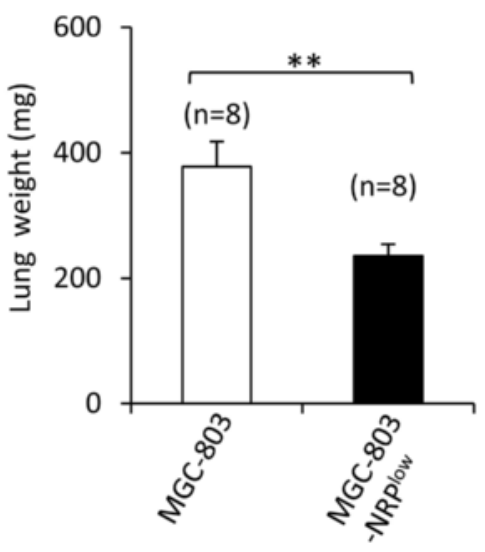

F

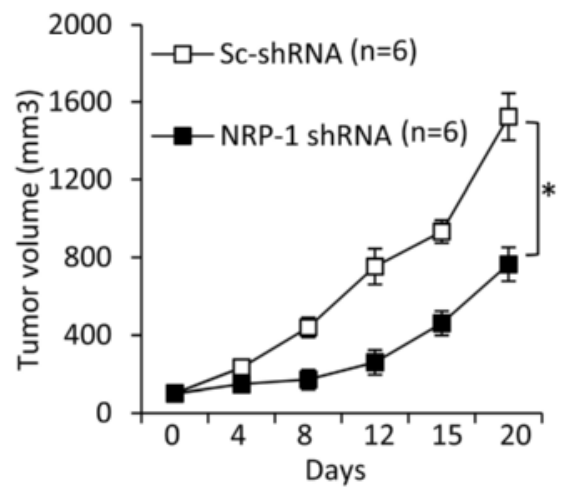

Fig. 4 Tumorigenesis, metastasis and tumor growth in vivo. $\mathbf{a}$, b MGC-803 and MGC-803-NRPlow cells were subcutaneously inoculated into the mice. a Tumor volumes were recorded. The number of tumor-bearing mice/ total number of mice are shown in brackets. $\mathbf{b}$ Tumors were weighed at the end of experiments. $\mathbf{c}$, $\mathbf{d}$ The above cells were intravenously injected into the mice. The mice were killed 7 weeks later to harvest the lungs, which were sectioned and stained by hematoxylin/eosin (c), and weighed (d). e, f BGC823 tumors were established in mice, and injected with control Sc-shRNA or NRP-1 shRNA when they reached $\sim 100 \mathrm{~mm}^{3}$ in volume. e lllustrated are representative tumor sections prepared 4 days following intratumoral injection. NRP-1 was immunostained brown by an anti-NRP-1 Ab. Magnification $\times 400$. $\mathbf{f}$ The sizes of tumors were measured. " $n$ " indicates the number of tumors. "**" indicates $P<0.05$, and "**", $P<0.001$ 


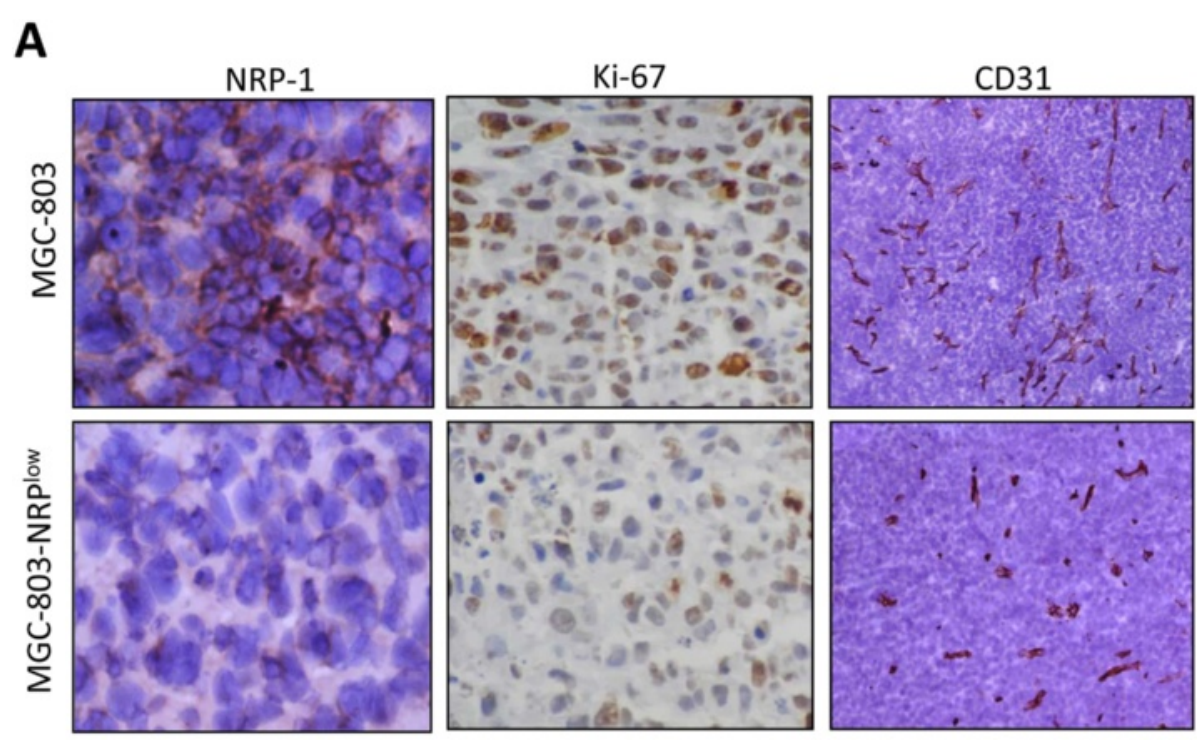

B
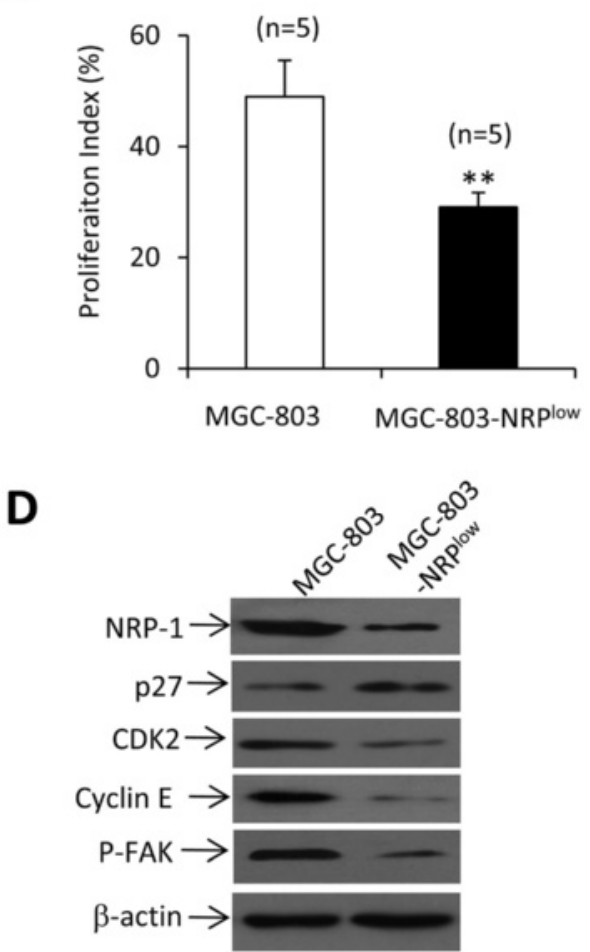

C

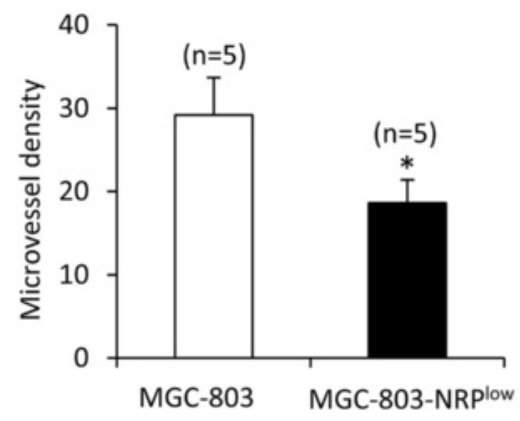

Fig. 5 Tumoral angiogenesis and cell proliferation in situ. a Illustrated are representative sections prepared from tumors in Fig. 4b. Sections were stained with antibodies against NRP-1 (left panel), Ki67 (middle panel) or CD31 (right panel). The cell proliferation index (b) and microvessel density (c) were quantified. " $n$ " indicates the number of tumors examined. $\mathbf{d}$ Homogenates prepared from tumors in Fig. $4 b$ were immunoblotted. The density of each band was measured and normalized to that of $\beta$-actin. "**" $(P<0.05)$ and "**" $(P<0.001)$ indicate a significant difference from MGC-803 tumors

siRNAs targeting VEGFR2, EGFR and c-Met, and then incubated with recombinant human VEGF-165, EGF and HGF proteins, respectively. Transfection of siRNAs targeting VEGFR2, EGFR and c-Met resulted in markedly downregulation of VEGFR2, EGFR and c-Met, but had no effect on the expression of NRP-1, in both MGC-803 and MGC-803-NRP ${ }^{\text {low }}$ cells (Fig. 6a-c).

Incubation of recombinant VEGF-165 protein had no effect on the expression of NRP-1 or VEGFR2, but markedly increased the levels of P-FAK, a downstream 


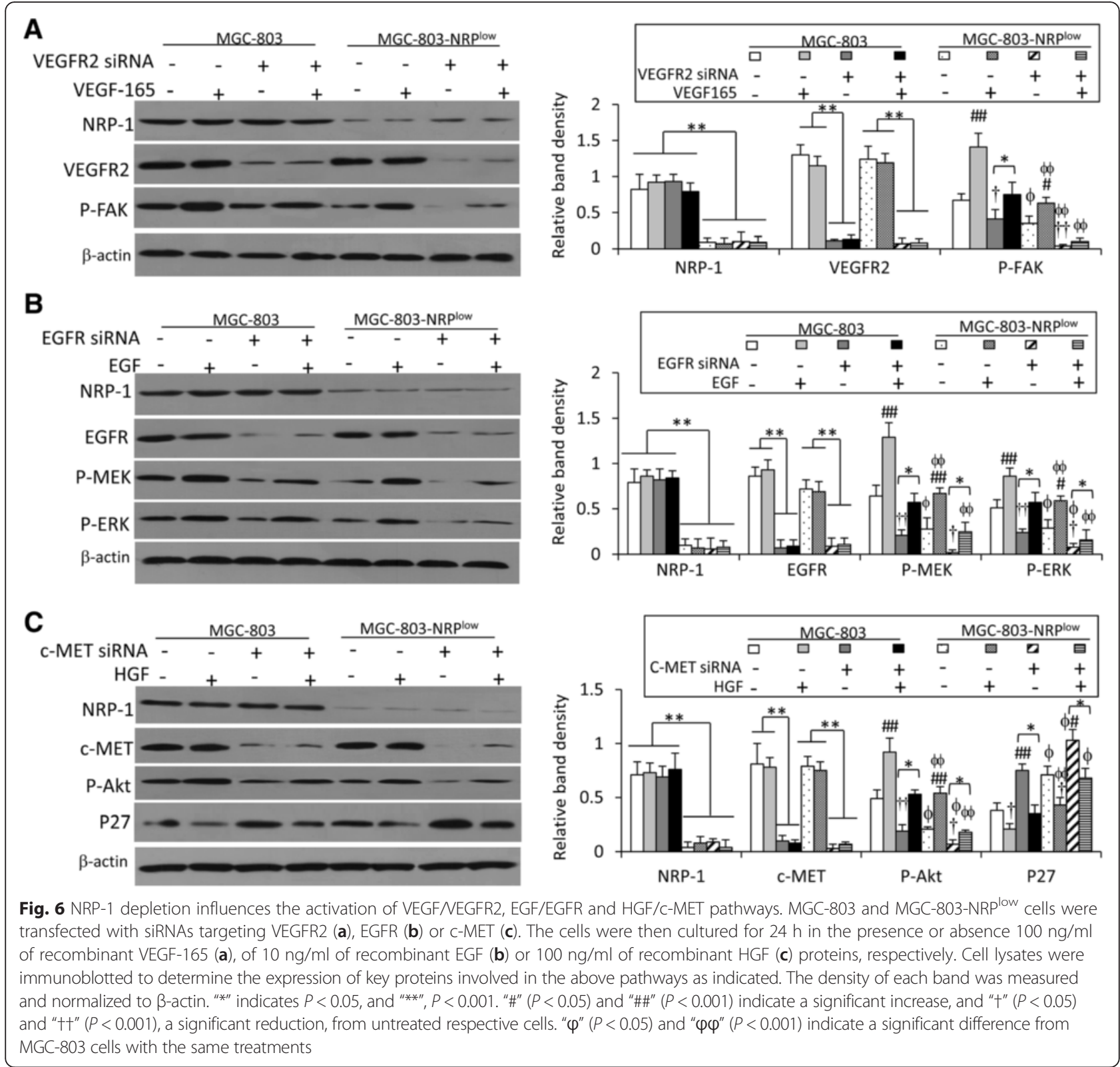

factor of VEGFR2 pathways [11], in MGC-803 and MGC803-NRP ${ }^{\text {low }}$ cells (Fig. 6a). Transfection of VEGFR2 siRNA significantly reduced the level of P-FAK in MGC-803 and MGC-803-NRP $^{\text {low }}$ cells (Fig. 6a). The level of P-FAK was significantly lower in MGC-803-NRP ${ }^{\text {low }}$ cells than MGC803 cells, when they received the same treatments, either untreated, incubation with VEGF-165 protein, transfection with VEGFR2 siRNA, or the combination (Fig. 6a).

NRP-1 regulates the EGFR signaling pathways, resulting in activation of downstream signaling cascades including the MEK/ERK pathway [13]. Here we showed that incubation of recombinant EGF protein had no effect on the expression of NRP-1 or EGFR, but increased the levels of PMEK and P-ERK proteins in parental MGC-803 and
MGC-803-NRP ${ }^{\text {low }}$ cells (Fig. 6b). EGFR siRNA transfection significantly inhibited the expression of P-MEK and P-ERK in parental MGC-803 cells and MGC-803-NRP low cells (Fig. 6b). The levels of P-MEK and P-ERK were significantly lower in MGC-803-NRP ${ }^{\text {low }}$ cells than MGC-803 cells, when they received the same treatments, either untreated, incubation with EGF protein, transfection with EGFR siRNA, or the combination (Fig. 6b).

The HGF/c-Met pathway is involved in the progression of gastric cancer by activating Akt pathways [18]. Here we demonstrated that incubation of recombinant HGF protein had no effect on the expression of NRP-1 or c-Met, but increased the levels of P-Akt and reduced the levels of p27 in parental MGC-803 and MGC-803-NRP ${ }^{\text {low }}$ cells (Fig. 6c). 
Transfection of c-Met siRNA significantly downregulated the expression of P-Akt but increased the expression of p27 in parental MGC-803 cells and MGC-803-NRP $P^{\text {low }}$ cells (Fig. 6c). The levels of P-Akt were significantly lower, while the levels of p27 were significantly higher, in MGC803-NRP ${ }^{\text {low }}$ cells than MGC-803 cells, when they received the same treatments, either untreated, incubation with HGF protein, transfection with c-Met siRNA, or the combination (Fig. 6c).

\section{Discussion}

NRP-1 has been shown to participate in the proliferation of cells from hepatocellular carcinoma [2], lung cancer [6], leukemia and lymphoma [8] and medulloblastoma [9]. In accord, the present results have demonstrated that depletion of NRP-1 inhibited the proliferation of gastric cancer cells by inducing cell cycle arrest in the G1/S phase through upregulating p27 and downregulating cyclin E and CDK2. P27 is implicated in the negative regulation of cell cycle progression from $G_{1}$ to $S$ phase by binding to and modulating the activity of CDKs [24]. Conversely, the cyclin $\mathrm{E} / \mathrm{CDK} 2$ complex promotes progression from $\mathrm{G}_{1}$ to $\mathrm{S}$ phase by triggering the initiation of DNA replication [24].
Distant metastasis is the leading cause of mortality for gastric cancer, and is a major treatment obstacle [21]. Antiangiogenic therapy, such as VEGF inhibition, continues to be a focus for developing anticancer drugs for gastric cancer [16]. However, VEGF inhibition demonstrates only transient and invariable benefits, and can also induce hypertension and renal toxicity due to its off-target effects by blocking the cellular signaling of VEGFR1 [26]. The recognition that NRP-1 is engaged by specific VEGF isoforms has expanded the landscape for developing modulators of VEGF-dependent signaling [27]. Here we showed that NRP-1 depletion inhibited VEGF-activated VEGF/VEGFR2 pathway, which is crucial for tumor angiogenesis by regulating the phosphorylation of FAK [28], a key factor in cell migration and metastasis [29]. In support of our results, blockage of NRP-1 inhibited tumor growth and VEGFmediated angiogenesis [11]. The VEGF/NRP-1 pathway is also involved in cancer cell proliferation by activating Akt, leading to sequential p27 downregulation [30].

NRP-1 interacts with EGFR and promotes its signaling cascade elicited upon EGF stimulation in cancer cells [13]. The EGF/EGFR pathway is involved in the progression of gastric cancer [17]. In accord, the present study has

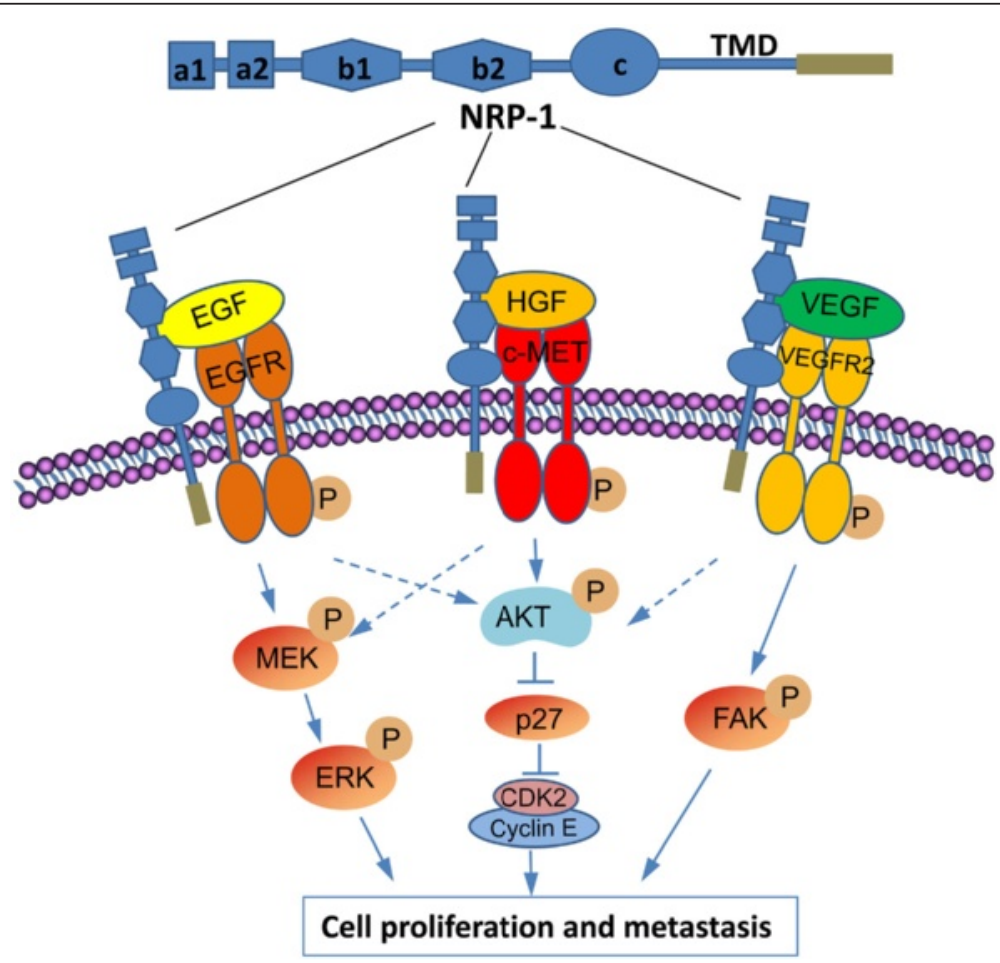

Fig. 7 NRP-1 structure and a proposed model of interactions of NRP-1 as multifunctional co-receptors with VEGF/NEGFR2, EGF/EGFR and HGF/c-MET pathways in gastric cancer cells. NRP-1 molecule consists of five extracellular domains, a single-pass transmembrane domain (TMD) and a short cytosolic tail. " $\rightarrow$ " indicates promotion, positive regulation or activation; " $\perp$ ", inhibition, negative regulation or blockade. A dotted line indicates uncertain mechanisms, while a solid line, identified mechanisms. Abbreviations: CDK, cyclin-dependent kinase; EGF, epithelial growth factor; EGFR, epithelial growth factor receptor; ERK, extracellular-signal-regulated kinase; FAK, focal adhesion kinase; HGF, hepatocyte growth factor; MEK, mitogen-activated protein kinase; NRP-1, neuropilin-1; VEGF, vascular endothelial growth factor; VEGFR, vascular endothelial growth factor receptor 
demonstrated that NRP-1 silencing counteracted ligandinduced EGFR activation, evidenced by the reduced phosphorylation of MEK and ERK, two downstream factors of the EGFR pathway [31]. The MEK/ERK pathway participates in the progression of gastric cancer by contributing to cell proliferation and metastasis $[17,32]$.

NRP-1 is also implicated in the activation of HGFinduced signaling and cellular responses in cancer cells [14]. The involvement of $\mathrm{HGF} / \mathrm{c}-$ Met pathway leads to the activation of PI3K/Akt signaling pathway in gastric cancer cells [18]. Accordingly, we have demonstrated herein that NRP-1 depletion attenuated HGF-stimulated the activation of HGF signaling pathway, evidenced by reduced activation of Akt.

The general domain structure of NRP-1 [1] and proposed mechanisms for its role as co-receptors in the proliferation and metastasis of gastric cancer cells are summarized in Fig. 7. NRP-1 co-interacts with VEGF/VEGFR2 [10-12], EGF/EGFR [13] and HGF/c-Met [14] pathways. The activation of the VEGFR2 pathway results in the phosphorylation of FAK, which promotes cell metastasis [11, 28]. The activation of the c-Met pathway induces sequential activation of Akt [25], which in turn leads to downregulation of p27 [24, 33]. P27 negatively regulates cell cycle progression by modulating the activity of CDK2/cyclin E complex [24]. The activation of EGFR pathway leads to the phosphorylation of MEK and ERK, which contributes to the proliferation and metastasis of gastric cancer cells [17, 32]. Although not investigated in the present study, the cross-talks among the above signaling pathways may also be involved in cancer cell proliferation and metastasis [31].

\section{Conclusions}

In summary, NRP-1 is overexpressed in gastric cancer tissues, and its expression correlates with the clinical staging, tumor differentiation and pathological types of gastric cancer. NRP-1 depletion inhibited the proliferation and migration of gastric cancer cells, and suppressed their ability to form tumors and to metastasize to lungs, and therapeutic NRP-1 shRNA inhibited the growth of established gastric tumors in experimental animals. NRP-1 depletion counteracted the activation of VEGF/VEGFR2, EGF/EGFR and HGF/c-Met pathways stimulated by respective ligands, indicating that NRP-1 acts as multifunctional co-receptors in gastric cancer cells. Taken together, the present results suggest that NRP-1 may be a valuable biomarker and potential therapeutic target for gastric cancer, particularly for those expressing higher levels of NRP-1.

\section{Additional file}

Additional file 1: Supplementary materials. (DOCX $23 \mathrm{~kb})$

\section{Abbreviations}

ANOVA: Analysis of variance; BrdU: Bromodeoxyuridine; CDK: Cyclin-dependent kinase; EGF: Epithelial growth factor; EGFR: Epithelial growth factor receptor; ERK: Extracellular-signal-regulated kinase; FAK: Focal adhesion kinase; FBS: Fetal bovine serum; HGF: Hepatocyte growth factor; MEK: Mitogen-activated protein kinase; NRP-1: Neuropilin-1; qRT-PCR: Quantitative reverse-transcription polymerase chain reaction; VEGF: Vascular endothelial growth factor; VEGFR: Vascular endothelial growth factor receptor.

\section{Competing interests}

The authors declare that they have no competing interests.

\section{Authors' contributions}

LL collected the clinical specimens, performed immunohistochemistry, analyzed the data and drafted the manuscript. XJ performed cell culture, immunoblotting, RT-PCR, cell growth and migration assays and animal experiments. QZ participated in the immunohistochemistry and statistical analysis. XD participated in cell culture and assays, and animal experiments. YG assisted in collecting and processing clinical specimens. YH participated in sample handling, storage and collection of the clinical data. $\mathrm{HQ}$ assisted in cell and animal experiments. FX contributed in study design and manuscript revision. XS and XX designed the project, supervised the study and finalized the manuscript. All authors read and approved the final manuscript.

\section{Acknowledgements}

This work was supported by grants from the National Natural Scientific Foundation of China (81272467 and 81472321), Bureau of Science and Technology of Qingdao City (2013Y005), Heilongjiang Provincial Scientific Fund for Youths (QC2013C098), and Qingdao Outstanding Health Professional Development Fund (YQ2014Y14), China. H. Lin and X. Jiang contributed equally to this work.

\section{Author details}

${ }^{1}$ Department of General Surgery, Qingdao Municipal Hospital, Qingdao 266011, China. ${ }^{2}$ Department of General Surgery, the First Affiliated Hospital of Harbin Medical University, No. 23 Youzheng Street, Harbin 150001, China. ${ }^{3}$ Department of Obstetrics and Gynecology, Qingdao Municipal Hospital, Qingdao 266011, China. ${ }^{4}$ Department of Gastroenterology, Qingdao Municipal Hospital, No. 1 Jiaozhou Road, Qingdao 266011, China.

Received: 19 October 2015 Accepted: 11 January 2016

Published online: 22 January 2016

\section{References}

1. Prud'homme GJ, Glinka Y. Neuropilins are multifunctional coreceptors involved in tumor initiation, growth, metastasis and immunity. Oncotarget. 2012:3:921-39.

2. Bergé M, Allanic D, Bonnin P, de Montrion C, Richard J, Suc M, et al. Neuropilin-1 is upregulated in hepatocellular carcinoma and contributes to tumour growth and vascular remodelling. J Hepatol. 2011;55:866-75.

3. Staton CA, Koay I, Wu JM, Hoh L, Reed MW, Brown NJ. Neuropilin-1 and neuropilin-2 expression in the adenoma-carcinoma sequence of colorectal cancer. Histopathology. 2013;62:908-15.

4. Alattar M, Omo A, Elsharawy M, Li J. Neuropilin-1 expression in squamous cell carcinoma of the oesophagus. Eur J Cardiothorac Surg. 2014;45:514-20.

5. Pan Q, Chanthery Y, Liang WC, Stawicki S, Mak J, Rathore N, et al. Blocking neuropilin-1 function has an additive effect with anti-VEGF to inhibit tumor growth. Cancer Cell. 2007;11:53-67.

6. Jia H, Cheng L, Tickner M, Bagherzadeh A, Selwood D, Zachary I. Neuropilin1 antagonism in human carcinoma cells inhibits migration and enhances chemosensitivity. Br J Cancer. 2010;102:541-52.

7. Nasarre C, Roth M, Jacob L, Roth L, Koncina E, Thien A, et al. Peptide-based interference of the transmembrane domain of neuropilin-1 inhibits glioma growth in vivo. Oncogene. 2010;29:2381-92.

8. Karjalainen K, Jaalouk DE, Bueso-Ramos CE, Zurita AJ, Kuniyasu A, Eckhardt $\mathrm{BL}$, et al. Targeting neuropilin-1 in human leukemia and lymphoma. Blood. 2011;117:920-7.

9. Snuderl M, Batista A, Kirkpatrick ND, Ruiz de Almodovar C, Riedemann L, Walsh EC, et al. Targeting placental growth factor/neuropilin 1 pathway inhibits growth and spread of medulloblastoma. Cell. 2013;152:1065-76. 
10. Hamerlik P, Lathia JD, Rasmussen R, Wu Q, Bartkova J, Lee M, et al. Autocrine VEGF-VEGFR2-Neuropilin-1 signaling promotes glioma stem-like cell viability and tumor growth. J Exp Med. 2012;209:507-20.

11. Herzog B, Pellet-Many C, Britton G, Hartzoulakis B, Zachary IC. VEGF binding to NRP1 is essential for VEGF stimulation of endothelial cell migration, complex formation between NRP1 and VEGFR2, and signaling via FAK Tyr407 phosphorylation. Mol Biol Cell. 2011;22:2766-76.

12. Barr MP, Gray SG, Gately K, Hams E, Fallon PG, Davies AM, et al. Vascular endothelial growth factor is an autocrine growth factor, signaling through neuropilin-1 in non-small cell lung cancer. Mol Cancer. 2015;14:45.

13. Rizzolio S, Rabinowicz N, Rainero E, Lanzetti L, Serini G, Norman J, et al. Neuropilin-1-dependent regulation of EGF-receptor signaling. Cancer Res. 2012;72:5801-11.

14. Matsushita A, Götze T, Korc M. Hepatocyte growth factor-mediated cell invasion in pancreatic cancer cells is dependent on neuropilin-1. Cancer Res. 2007:67:10309-16.

15. Akagi M, Kawaguchi M, Liu W, McCarty MF, Takeda A, Fan F, et al. Induction of neuropilin-1 and vascular endothelial growth factor by epidermal growth factor in human gastric cancer cells. Br J Cancer. 2003;88:796-802.

16. Ilson DH. Angiogenesis in gastric cancer: hitting the target? Lancet. 2014; 383:4-6.

17. Nagatsuma AK, Aizawa M, Kuwata T, Doi T, Ohtsu A, Fujii H, et al. Expression profiles of HER2, EGFR, MET and FGFR2 in a large cohort of patients with gastric adenocarcinoma. Gastric Cancer. 2015;18:227-38.

18. Hao NB, Tang B, Wang GZ, Xie R, Hu CJ, Wang SM, et al. Hepatocyte growth factor (HGF) upregulates heparanase expression via the PI3K/Akt/NF-KB signaling pathway for gastric cancer metastasis. Cancer Lett. 2015;361:57-66.

19. Jemal A, Bray F, Center MM, Ferlay J, Ward E, Forman D. Global cancer statistics. CA Cancer J Clin. 2011;61:69-90.

20. Bang YJ, Van Cutsem E, Feyereislova A, Chung HC, Shen L, Sawaki A, et al. Trastuzumab in combination with chemotherapy versus chemotherapy alone for treatment of HER2-positive advanced gastric or gastrooesophageal junction cancer (ToGA): a phase 3, open-label, randomized controlled trial. Lancet. 2010;376:687-97.

21. Hu B, El Hajj N, Sittler S, Lammert N, Barnes R, Meloni-Ehrig A. Gastric cancer: classification, histology and application of molecular pathology. J Gastrointest Oncol. 2012:3:251-61.

22. Sun $X P$, Dong $X$, Lin $L$, Jiang $X$, Wei $Z$, Zhai $B$, et al. Up-regulation of survivin by AKT and hypoxia-inducible factor 1a contributes to cisplatin resistance in gastric cancer. FEBS J. 2014;281:115-28.

23. Wei Z, Jiang X, Qiao H, Zhai B, Zhang L, Zhang Q, et al. STAT3 interacts with Skp2/p27/p21 pathway to regulate the motility and invasion of gastric cancer cells. Cell Signal. 2013;25:931-8.

24. Wander SA, Zhao D, Slingerland JM. p27: a barometer of signaling deregulation and potential predictor of response to targeted therapies. Clin Cancer Res. 2011;17:12-8.

25. Kawakami H, Okamoto I, Arao T, Okamoto W, Matsumoto K, Taniguchi H, et al. MET amplification as a potential therapeutic target in gastric cancer. Oncotarget. 2013:4:9-17.

26. Hayman SR, Leung N, Grande JP, Garovic VD. VEGF inhibition, hypertension, and renal toxicity. Curr Oncol Rep. 2012;14:285-94.

27. Djordjevic S, Driscoll PC. Targeting VEGF signalling via the neuropilin co-receptor. Drug Discov Today. 2013;18:447-55.

28. Jean C, Chen XL, Nam JO, Tancioni I, Uryu S, Lawson C, et al. Inhibition of endothelial FAK activity prevents tumor metastasis by enhancing barrier function. J Cell Biol. 2014;204:247-63.

29. Alanko J, Mai A, Jacquemet G, Schauer K, Kaukonen R, Saari M, et al. Integrin endosomal signalling suppresses anoikis. Nat Cell Biol. 2015;17(11):1412-21. doi:10.1038/ncb3250

30. Yoshida A, Shimizu A, Asano H, Kadonosono T, Kondoh SK, Geretti E, et al. VEGF-A/NRP1 stimulates GIPC1 and Syx complex formation to promote RhoA activation and proliferation in skin cancer cells. Biol Open. 2015;4:1063-76.

31. Chakraborty S, Li L, Puliyappadamba VT, Guo G, Hatanpaa KJ, Mickey B, et al. Constitutive and ligand-induced EGFR signalling triggers distinct and mutually exclusive downstream signalling networks. Nat Commun. 2014;5:5811.

32. Fujimori Y, Inokuchi M, Takagi Y, Kato K, Kojima K, Sugihara K. Prognostic value of RKIP and p-ERK in gastric cancer. J Exp Clin Cancer Res. 2012;31:30.

33. Shin I, Yakes FM, Rojo F, Shin NY, Bakin AV, Baselga J, et al. PKB/Akt mediates cell-cycle progression by phosphorylation of p27(Kip1) at threonine 157 and modulation of its cellular localization. Nat Med. 2002;8:1145-52.

\section{Submit your next manuscript to BioMed Central and we will help you at every step:}

- We accept pre-submission inquiries

- Our selector tool helps you to find the most relevant journal

- We provide round the clock customer support

- Convenient online submission

- Thorough peer review

- Inclusion in PubMed and all major indexing services

- Maximum visibility for your research

Submit your manuscript at www.biomedcentral.com/submit
Biomed Central 